\title{
USO DE METILFENIDATO ENTRE ACADÊMICOS NO INTERIOR DE RONDÔNIA
}

\author{
Mariane Suelen Izidoro ALBERTO ${ }^{1}$ \\ Tiago Barcelos VALIATTI ${ }^{2}$ \\ Izabel Bárbara BARCELOS ${ }^{3}$ \\ Jeferson de Oliveira SALVI ${ }^{4}$
}

\begin{abstract}
${ }^{1}$ Farmacêutica, graduada pelo Centro Universitário Luterano de Ji-Paraná. marianealberto@gmail.com
${ }^{2}$ Graduando em Farmácia pelo Centro Universitário Luterano de Ji-Paraná. tiago_valiatti@ hotmail.com

${ }^{3}$ Graduanda em Farmácia pelo Centro Universitário Luterano de Ji-Paraná. izabelbbarcelos@gmail.com

${ }^{4}$ Farmacêutico, especialista, docente do curso de Farmácia do Centro Universitário Luterano de Ji-Paraná. jefersonsalvi@hotmail.com
\end{abstract}

\section{Recebido em: 03/05/2016 - Aprovado em: 10/03/2017 - Disponibilizado em: 01/07/2017}

\begin{abstract}
RESUMO
Nos últimos anos é visível o aumento no consumo de medicamentos psicotrópicos, dentre eles o metilfenidato, que em muitos casos são utilizados por universitários afim de melhorar o aprendizado. O presente estudo teve como objetivo caracterizar o perfil do uso de metilfenidato entre acadêmicos de um centro universitário localizado no interior do estado de Rondônia. Constituíram o estudo 150 acadêmicos oriundos dos cursos de biomedicina, enfermagem e farmácia. Houve predominância do sexo feminino, a maior parte dos entrevistados já haviacursado a disciplina de farmacologia, no entanto apenas $48 \%$ souberam definir medicamentos psicotrópicos. Com relação ao uso de metilfenidato $18 \%$ afirmaram já tê-lo praticado, sendo que desses $45 \%$ o adquiriram com receita médica. O principal motivo de uso foi para melhoria do aprendizado (85\%), onde a maioria dos acadêmicos afirmou terem alcançado o efeito desejado, apesar de 59\% relataram efeitos adversos. Os dados obtidos evidenciam a utilização do metilfenidato para obtenção de melhora no desempenho estudantil, tendência observada em outros estudos, que tem trazido preocupação no contexto de Saúde Pública.
\end{abstract}

Palavras-chave: Psicotrópicos. Universitários. Metilfenidato. Uso irracional. Aprimoramento cognitivo.

\begin{abstract}
In recent years visible is the increase in consumption of psychotropic drugs, including methylphenidate, which in many cases are used by students to improve learning. This study aimed to characterize the profile of the use of methylphenidate among academics of a university located in the state of Rondonia. They constituted the study 150 students coming from biomedicine courses, nursing and pharmacy. Patients were predominantly female, the majority of respondents had taken the course of pharmacology, however only $48 \%$ could define psychotropic medications. Regarding the use of methylphenidate $18 \%$ said they already have it practiced, and of these $45 \%$ have purchased it with a prescription. The main reason for usage was to improve learning $(85 \%)$, where most of the scholars said they have achieved the desired effect, while 59\% reported adverse effects. The data show the use of methylphenidate to obtain improvement in student performance, trend observed in other studies, which have brought concern the public health context.
\end{abstract}

Keywords: Psychotropics. University.Methylphenidate.Irrational Use. CognitiveImprovement.

\section{INTRODUÇÃO}

Psicotrópicos atuam de maneira seletiva sobre o Sistema Nervoso Central (SNC) promovendo modificações no comportamento e no humor. No Brasil, a dispensação dessa classe só pode ser realizada perante apresentação da receita, pois está sujeita ao controle especial, porém o que se observa nos últimos anos, é o aumento do consumo desses medicamentos, que em 
muitas vezes são adquiridos sem prescrição médica (Firmo et al., 2013; Da Silva e Iguti, 2013; Tsuda e Christoff, 2015; Shirakawa et al., 2012).

Dentre os que fazem o uso de substâncias psicotrópicas, estão os alunos do ensino superior que as utilizam para elevar seu rendimento. Observa-se a cada ano o aumenta no consumo dessas substâncias por esse grupo populacional, promovendo uma série de discussões, pois o uso indevido dessas pode acarretar perturbações mentais, estado de pânico e alucinações, além de possuírem potencial para o desenvolvimento de dependência no usuário (Bruntonet al., 2012;Pasquini, 2013).

Entre as substâncias psicotrópicas comumente utilizadas pelos acadêmicos, destaca-se uma droga proveniente da anfetamina, o Metilfenidato, indicado para o tratamento de Transtorno do Déficit de Atenção/Hiperatividade (TDAH). O fato do Metilfenidato promover sobre os receptores alfa e beta adrenérgico fortes efeitos agonistas, faz com que o SNC fique com significativo estado de alerta, ocasionando assim uma maior concentração, controle dos impulsos e melhor desempenho motor (Mota e Pessanha, 2014; Barros e Ortega, 2001).

O presente estudo objetivoucaracterizar o perfil do uso do metilfenidato entre os acadêmicos de um Centro Universitário localizado no interior do estado de Rondônia.

\section{METODOLOGIA}

Trata-se de um estudo transversal e exploratório, desenvolvido junto ao Centro Universitário Luterano de Ji-Paraná (CEULJI/ULBRA) como parte integrante de um projeto de extensão sobre farmacoepidemiologia e farmacovigilância de medicamentos psicotrópicos, aprovado pelo Comitê de Ética e Pesquisa sob o parecer 558.116 de 18 de agosto de 2014.

A população foi constituída por acadêmicos da área da saúde que possuíam disciplinas relacionadas à farmacologia na grade, o que incluiu os cursos de Biomedicina, Enfermagem e Farmácia. A amostra foi estratificada por curso, participando 50 indivíduos por grupo, perfazendo um total de 150 participantes.

$\mathrm{O}$ instrumento para coleta dos dados tratou-se de um questionário estratificadocom as alternativas nominais não paramétricas relacionadas à frequência: $\operatorname{sim}$ e não. As respostas foram codificadas em valores numéricos para análiseda distribuição das médias. O questionário foi entregue em sala de aula, após explanação dos objetivos e mediante a assinatura do Termo de Consentimento Livre e Esclarecido (TCLE).

Os dados coletados foram tabulados e analisados por estatística descritiva simples com auxílio do programa Microsoft Excel ${ }^{\circledR}$. As médias dos grupos foram comparadas por análise de variância (ANOVA) seguida pelo Teste deKruskall-Wallis analisado pelo 
software GraphPadPrism $^{\circledR}$ (versão 6.0). Os valores foram considerados significativos para $\mathrm{p}<0,05$.

RESULTADOS
A tabela 1 demonstra a frequência relativa dos gêneros por grupo, evidencia-se a prevalência dasmulheres entre os indivíduos que, de um modo geral, representaram $78 \%$ da totalidade da amostra.

Tabela 1-Caracterização dos participantes com base no gênero.

\begin{tabular}{cccc}
\hline Curso & Feminino (\%) & Masculino (\%) & Total (\%) \\
\hline Biomedicina & 76 & 24 & 100 \\
Enfermagem & 98 & 02 & 100 \\
Farmácia & 60 & 40 & 100 \\
\hline
\end{tabular}

A figura I apresenta o valor médio das cinco primeiras questões a partir da codificação realizada: $\operatorname{sim}=1$ e não $=2$, ou seja, quanto mais próximo de 1 as respostas são afirmativas.

Figura 1. Distribuição do valor médio obtido para algumas questões.

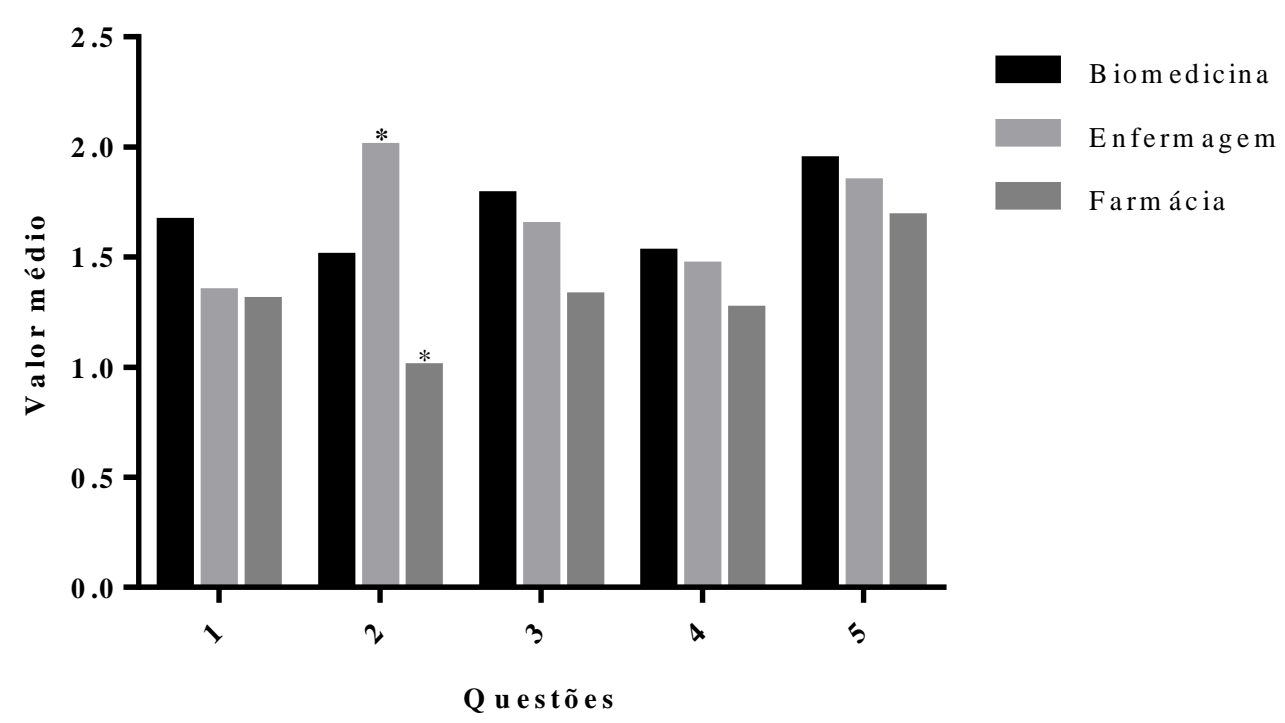

1. Já cursou farmacologia? 2. Você sabe o que é um psicotrópico? 3. Você sabe para que o metilfenidatoé indicado? 4. Conhece algum colega que já fez ou faz o uso do metilfenidato5. Você já fez uso desse medicamento? * p<0,05 (Teste de Tukey). ** Valores numéricos próximos a 1 correspondem às respostas afirmativas e a 2, respostas negativas.

A análise revela que a maioria dos entrevistados teve o contato com a disciplina de farmacologia, mas menos da metade (48\%) declararam definir psicotrópicos. Neste quesito observou-se uma diferença significativa entre os cursos de enfermagem e farmácia. 
A habilidade de relatar sobre a indicação do uso do metilfenidato foi menor do que o conhecimento de alguém que fez ou faz uso dele, muito embora, a frequência relativa dos que declarado tê-lo consumido represente apenas $18 \%$.
A tabela 2 descreve sobre a maneira da aquisição do fármaco individualizada por curso. De um modo geral, dentre os que responderam positivamente para o consumo, $45 \%$ declarouter obtido a droga legalmente por prescrição médica e $4 \%$ com outro profissional e51\% preferiu não responder.

Tabela 2. Distribuição da frequência relativa dos tipos de aquisição categorizada por curso.

\begin{tabular}{cccccc}
\hline Curso & Com receita & Sem receita & Com amigo & Com familiar & Total \\
\hline Biomedicina & $67 \%$ & $33 \%$ & $0 \%$ & $0 \%$ & $100 \%$ \\
Enfermagem & $25 \%$ & $0 \%$ & $75 \%$ & $0 \%$ & $100 \%$ \\
Farmácia & $38 \%$ & $6 \%$ & $50 \%$ & $6 \%$ & $100 \%$ \\
\hline
\end{tabular}

Quando indagados sobre o motivo do uso $85 \%$ declararam o ter realizado para melhorado aprendizado. Desses, a metade foi do curso de Farmácia. Sendo que, 10\% tiveram a curiosidade como proponente $\mathrm{e}$ apenas $5 \%$ para tratamento do déficit de atenção.

Com relação ao efeito desejado, $85 \%$

obtiveram o que esperavam do medicamento, ou seja, conseguiram aumentar a capacidade de concentração e aprendizado. Entretanto, a maioria (59\%)reconheceu a manifestação de efeitos adversos após o consumo do metilfenidato. A tabela 3 descreve a frequência dos tipos de reações adversas e as caracteriza por grupo.

Tabela 3. Frequência relativa dos principais efeitos adversos identificados.

\begin{tabular}{lllllll}
\hline Curso & Boca seca & Cefaleia & Insônia & Palpitação & Perda do apetite & Outro \\
\hline Biomedicina & 33 & 0 & 66 & 66 & $0 \%$ & $0 \%$ \\
Enfermagem & 12 & 37 & 12 & 25 & 12 & 37 \\
Farmácia & 30 & 18 & 31 & 25 & 6 & 12 \\
\hline
\end{tabular}

*Os valores variam conforme a totalidade dos sinais e sintomas, um mesmo participante marcoumais de uma opção.

A legalidade da aquisição como restrição ao uso foi abordada considerando a possível ausência de regulamentação. A pergunta: "Se o medicamento não fosse controlado, você usaria?" Obteve resposta positiva para $24 \%$ dos entrevistados de todos os cursos, 29\% declarou que mesmo assim não fariam uso e $45 \%$ optou pela opção de que talvez utilizasse. 


\section{DISCUSSÃO}

Atualmente verifica-se uma expansão no uso do metilfenidato, empregado no tratamento de patologias da atenção e para melhoria de funções cognitivas em indivíduos saudáveis, fato o qual têm gerado diversos debates em Saúde Pública. O consumo desse medicamento por pessoas saudáveis não diagnosticadas com TDAH é uma prática crescente em vários países tais como: Inglaterra, Canadá e Estados Unidos, caracterizando a automedicação com riscos dos danos mediante o uso irracional, neste caso, denominada de aprimoramento cognitivo farmacológico (Ortega et al, 2010).

Nos Estados Unidos a má utilização do metilfenidato para fins recreativos representa um desvio de finalidade que prevalece em cerca de $10 \%$ dos estudantes do ensino médio e até $35 \%$ dos estudantes universitários. Tal evidência pode estar associada à rapidez do início do efeito da droga e ao resultado que ela proporciona (Clemow, 2015).

Nesse contexto, diversos estudos têm avaliado o uso desse medicamento, Johansson et al (2016) sugerem o uso do metilfenidato à longo prazo para a redução da fadiga mental com melhora da cognição após acidentes cerebrais traumáticos. Vogelet al (2016) abordam o metilfenidato como candidato ao tratamento da dependência por cocaína, por apresentar efeitos subjetivos semelhantes e mais positivos em comparação com o opiáceo. Para Skoglundet al (2016) doses elevadas estão associadas ao aumento da adesão ao tratamento de longo prazo em casos de déficit de atenção.

Indivíduos que pertencem à área da saúde têm geralmente um maior conhecimento sobre medicamentos psicotrópicos (Brant; Carvalho, 2012), contudo observou-se no presente estudo que a menor parte dos entrevistados soube descrever corretamente a definição desses medicamentos, o que pode ser justificado pelo fato de os mesmos ainda estarem na graduação. Percentual ainda menor soube responder a finalidade do metilfenidato, sendo que entre os que souberam, a maioria pertencia ao curso de farmácia.

A utilização do metilfenidato foi identificada em um quinto da amostra de estudo, mas quase metade da amostra declarou conhecer algum colega que fez uso da substância. Resultados superiores foram encontrados por Pasquine (2013) que relatou o consumo em aproximados $45 \%$ dos universitários, sendo que $62,77 \%$ conheciam alguém que já fez ou faz uso. Neste mesmo estudo, entre aqueles que já fizeram uso de metilfenidato, a maioria declarou conhecimento acerca dos psicotrópicos. Tal evidência sugere que o conhecimento sobre os psicotrópicos esteja relacionadoao uso do fármaco. 
O uso do metilfenidato tem sido relacionado à busca por um melhor desempenho, como consequência da preocupação com o mercado de trabalho que exige maior preparação e atenção múltipla. Essa consideração refletiria no consumo precoce do metilfenidato como necessidade da conclusão da graduação, como também reflete na continuidade do uso desta substância que apresenta potencial para a dependência (Silva et al., 2012; Carvalho et al., 2014).

Os acadêmicos de biomedicina foram os que mais fizeram uso do medicamento com receita, verificando-se que entre os acadêmicos dos outros cursos houve maior uso indiscriminado. Outros estudos relatam o uso indiscriminado dessa substância, e ainda que o seu uso aumente progressivamente no decorrer dos períodos da graduação, possivelmente devido ao aumento do conteúdo a ser estudado (Carneiro et al, 2013; Cruz et al, 2011).

Segundo Bogle e Smith (2009) o consumo ilícito do metilfenidato entre universitários varia de 1,5 a 31\%, dependendo das características da amostra analisada. Cruz et al (2011) evidenciam que 7,5\% dos acadêmicos de medicina analisados em seu estudo sabem onde comprar esse medicamento sem receita, fato preocupante pois a notificação de receita deste medicamento é de difícil acesso, sendo que esse dado refletiria a realidade do Brasil quanto a frequente venda de medicamentos controlados de maneira ilícita.

DeSantiset al (2008) abordam que os universitários são os mais associados ao uso de metilfenidato para aprimorar seu desempenho, o que foi verificado no presente estudo, assim como no estudo de Pasquine (2013) onde todos os universitários afirmaram ter feito o uso para aprimorar o rendimento escolar. Ainda, a maioria alcançou o objetivo esperado, assim como no estudo de Carneiro et al (2013) onde a maior parte constatou melhora da concentração e do rendimento acadêmico.

Boa parte dos universitários informou ter apresentado efeitos colaterais, o que também foi verificado por Carneiro et al (2013), o autor aborda ainda que apesar dos efeitos colaterais os acadêmicos continuam fazendo uso devido aos benefícios encontrados.

$$
\text { Segundo Forlini e Racini (2009) }
$$
alguns autores defendem que o metilfenidato trata-se de uma droga segura, com poucos efeitos colaterais, e que a utilização para melhorar o desempenho seria louvável e pessoal, contudo outros autores consideram antiético e questionam os efeitos a longo prazo.

A segurança no tratamento cognitivo tem sido abordada em diferentes estudos, como node Kanget al (2016) onde o tratamento com metilfenidato se demonstrou eficaz na promoção da atenção e melhora do 
coeficiente intelectual de crianças, sem proporcionar algum distúrbio relacionado ao atraso de crescimento ou ao peso das mesmas.Kittel-Schneider et al (2016) em seu estudo não observaram efeitos citogenéticos relacionados à indicação para o tratamento do estresse crônico em adultos com transtornos da atenção.

Para os universitários estudados, em sua maioria, o fato desse medicamento ser ou não controlado, parece não exercer influência, possivelmente pelo fato de os mesmos levarem em consideração apenas os benefícios que buscam ao ingeri-lo. Barros e Ortega (2011) abordaram em seu estudo o metilfenidato e o aprimoramento cognitivo por meio deste, sendo que esse estudo tratouse de representações sociais que 20 universitários apresentaram sobre esse tema, onde os autores observaram que a questão mais levantada pelos entrevistados é a pressão social por resultados excelentes, onde os indivíduos são obrigados a realizar muitas tarefas em pouco tempo, o que estaria além de seus limites, sendo por isso a busca pelo uso de uma droga como o metilfenidato.

Dados da Comissão Internacional de Controle de Narcóticos abordam um aumento global da fabricação do metilfenidato. $\mathrm{O}$ Brasil apresenta crescente importação desse medicamento, passando de $578 \mathrm{~kg}$ importados em 2012 para $1820 \mathrm{~kg}$ importados em 2013, um aumento maior que 300\% (ONU, 2015). Shirakawa e Tejada (2012) destacam que o crescimento exponencial do consumo desse medicamento é preocupante devido ao seu uso indiscriminado.

De acordo com Caliman e Domitrovic (2013) nos últimos estudos promovidos pela Anvisa, o metilfenidato tem sido motivo de discussão, ganhado assim, destaque a média do consumo nacional desse medicamento, portanto, em um Boletim divulgado pela Anvisa (2012) sobre a prescrição e consumo do medicamento em questão nos anos de 2009 e 2011 destaca-se a seguinte frase: “ possíveis distorções na utilização de metilfenidato",

\section{CONCLUSÃO}

Constatou-se no presente estudo a tendência de uso do metilfenidato para melhora no desempenho entre universitários, sendo verificado o uso indiscriminado, o que gera grande preocupação com relação às consequências desse uso, como a dependência e efeitos colaterais. Além disso, reflete a falta de cumprimento da legislação vigente que determina o uso apenas sob prescrição médica. Esses dados sinalizam a importância dessa temática para Saúde Pública e a possibilidade de uma intervenção junto às disciplinas que envolvem ética e medicamentos.

\section{REFERÊNCIAS}

BRASIL. Boletim de Farmacoepidemiologia do SNGPC- Prescrição e consumo de metilfenidato no Brasil: Identificando riscos 
para o monitoramento e controle sanitário Ano II. N. 1. 2012 Agência Nacional de Vigilância Sanitária. Disponível em: http://www.anvisa.gov.br/sngpc/boletins/2012 /boletim_sngpc_2_2012_corrigido_2.pdf. [acesso em: 23 de abril de 2016].

BARROS, D.; ORTEGA, F. Metilfenidato e aprimoramento cognitivo farmacológico: representações sociais de universitários. Saúde e Sociedade, v. 20, n.2, p.350-362, 2011.

BOGLE, K.E.; SMITH, B.H. Illicit methylphenidate use: a review of prevalence, availability, pharmacology, and consequences. CurrentDrug Abuse Reviews, v.2, n.2, p.157-76, 2009.

BRANT, L.C.; CARVALHO, T.R.F. Metilfenidato: medicamento gadget da contemporaneidade. Interface, v.16, n.42, p. 623-36, 2012.

BRUNTON, L.L.; CHABNER, B.A.;KNOLLMAN, B.C. Goodman e Gilman: As Bases Farmacológicas da Terapêutica. 12 ed. Rio de Janeiro: Mcgraw Hill, 2012.

CALIMAN, L.V.; DOMITROVIC, N. Uma análise da dispensa pública do metilfenidato no Brasil: o caso do Espírito Santo. Physis

Revista de Saúde Coletiva. v.23, n.3, p.879882, 2013.

CARNEIRO, S.G.; PRADO, A.S.T.; MOURA, H.C.; STRAPASSON, J.F.; RABELO, N.F.; RIBEIRO, T.T.; JESUS, E.C. O uso não prescrito de metilfenidato entre acadêmicos de medicina.Cadernos Unifoa, n.1, p.53-59, 2013.

CARVALHO, T.RF.; BRANT, L.C.; MELO, M.B. Exigências de produtividade na escola e no trabalho e o consumo de metilfenidato.

Revista educação e sociedade, v. 35, n.127, p. 587-604, 2014.

CLEMOW, D.B. Misuseofmethylphenidate.

Current Topics in Behavioral

Neurosciences, 2015.
CRUZ, T. C.S.C. et al. Uso não prescrito de metilfenidato entre estudantes de medicina da Universidade Federal da Bahia. Gazeta

Médica da Bahia, v.81, n.1, p.3-6, 2011.

DA SILVA, T.O.;IGUTI, A.M.

Medicamentos Psicotrópicos Dispensados em Unidade Básica de Saúde em Grande Município do Estado de São Paulo.

RevistaEletrônicaGestão e Saúde, p.20042015, 2013.

DESANTIS, A.D.; WEBB, E.M.; NOAR, S.M. Illicit use of prescription ADHD medications on a college campus: a multimethodological approach. Journal of American College Health, v.57, p. 315-324, 2008.

FIRMO, W.C.A.; PAREDES, A.O.; CUNHA, C.L.F.; TORRES, A.G.; BUCCINI, D.F.

Análise das prescrições médicas de psicotrópicos de uma farmácia comercial no município de Bacabal, Maranhão. Journal of Management \& Primary Health Care, v.4, n.1, p.10-18, 2013.

FORLINI, C.; RACINE, E. Disagreements with implications: diverging discourses on the ethics of non-medical use of methylphenidate for performance enhancement. BMC Medical Ethics, v.10, n.9, 2009.

JOHANSSON, B.; WENTZEL, A.P.; ANDRÉLL, P.; RONNBACK, L.;

MANNHEIMER, C. Long-term treatment with methylphenidate for fatigue after traumatic brain injury.

ActaNeurologicaScandinavica, 2016.

KANG, K.D.; YUN S.W.; CHUNG, U.; KIM, T.H.; PARK, J.K.; PARK, I.H.; HAN, D.H. Effects of methylphenidate on body index and physical fitness in Korean children with attention deficit hyperactivity disorder.

Human psychopharmacology, v.31, n.2, p. 76-82, 2016.

KITTEL-SCHNEIDER, S. et al. Cytogenetic Effects of Chronic Methylphenidate Treatment and Chronic Social Stress in 
Adults with Attention-Deficit/Hyperactivity Disorder. Pharmacopsychiatry, 2016.

MOTA, J.S.; PESSANHA, F.F. Prevalência do uso de metilfenidato por universitários de campos dos Goytacazes. Vértices. v.16, n.1, p. $77-86,2014$

ORTEGA, F.; BARROS, D.; CALIMAN, L.; ITABORAHY, C.; JUNQUEIRA, L.; FERREIRA, C.P. A ritalina no Brasil: produções, discursos e práticas. Interface, v.14, n.34, p. 499-510, 2010.

PASQUINI, N.C. Uso de metilfenidato (MFD) por estudantes universitários com intuito de 'turbinar' o cérebro.Revista biologia e farmácia, v.9, n.2, p. 107-113, 2013.

SHIRAKAWA, D.M.; TEJADA, S.N.; MARINHO, C.A.F. Questões atuais no uso indiscriminado do metilfenidato. Omnia Saúde, v.9, n.1, p.46-53, 2012.

SILVA, A.C.P.; LUIO, C.A.; SANTOS, K.Y.P.; YASUI, S.; DIONISIO, G.H.L. A exposição do consumo de ritalina. Revista de Psicologia da UNESP, v.11, n.2, p. 44-57, 2012.
SKOGLUND, C.; BRANDT, L.; ALMQVIST, C.; D'ONOFRIO, B.M.; KONSTENIUS, M.; FRANCK, J.; LARSSON, H. Factors Associated With Adherence to Methylphenidate Treatment in Adult Patients With AttentionDeficit/Hyperactivity Disorder and Substance Use Disorders.

JournalofClinicalPsychopharmacology, 2016.

TSUDA, C.A.; CHRISTOFF, A.O. Avaliação do padrão de uso de estimulantes em uma faculdade de Curitiba - PR. Cadernos da Escola de Saúde,v.13, n.1, p. 116-132, 2015.

UNITED NATIONS.Report of the International Narcotics Control Board for 2014. New York: International Narcotics Control Board, 2015. Disponívelem: https://www.incb.org/documents/Publications /AnnualReports/AR2014/English/AR_2014.p df.[acesso em 10/04/2016].

VOGEL, M.; BUCHER, P.; STRASSER, J.; LIECHTI, ME.; KRAHENBUHL, S.; DURSTELER, K.M. Similar and Different? Subjective Effects of Methylphenidate and Cocaine in Opioid-Maintained Patients.JournalofPsychoactiveDrugs, v.3, p.1-8, 2016. 